\title{
Comparative study between double inversion recovery (DIR) and fluid- attenuated inversion recovery (FLAIR) MRI sequences for detection of cerebral lesions in multiple sclerosis
}

Shaima Fattouh Elkholy ${ }^{1 *}$, Marianne Abdallah Sabet ${ }^{1}$, Mohammad Edrees Mohammad² and Ramy Edward Ibrahim Asaad ${ }^{1}$

\begin{abstract}
Background: Multiple sclerosis (MS) is a common chronic inflammatory demyelinating disorder more common in young adults. MS is characterized mainly with white matter (WM) affection; however, considerable gray matter (GM) involvement is also noted in many patients. MRI is used for diagnosis and follow up of the disease using different pulse sequences; FLAIR imaging provides the highest sensitivity in the detection of supratentorial, juxtacortical, and the periventricular lesions but is less sensitive in the posterior fossa. A double inversion recovery (DIR) pulse sequence was recently introduced to improve the visibility of GM lesions and especially cortical lesions. The aim of this study is to assess the role of DIR sequence in the detection of brain lesions in patients with MS compared to FLAIR sequence.

Results: DIR showed a significantly higher number of MS lesions in infratentorial region ( $2.9 \pm 0.4$ compared to $2.25 \pm$ 0.3 in FLAIR) with a statistically significant difference $(p=0.002)$ and also in supratentorial periventricular regions (11.84 \pm 8.07 in DIR and $11.31 \pm 8.07$ in FLAIR, $p<0.001)$. DIR imaging also demonstrated significantly more intracortical lesions (7.12 \pm 1.2 compared to $1.4 \pm 0.9$ in FLAIR imaging) with a statistically significant difference $(p<0.001)$. On the other hand, corpus callosum lesions were significantly higher on FLAIR $(0.84 \pm 0.1)$ with respect to DIR imaging (0.68 \pm 0.1$)$ with a statistically significant difference in between $(p=0.025)$.

Conclusion: DIR is a powerful conventional MRI sequence for visualization of brain lesions in patients with MS and is superior to FLAIR sequence in detecting lesions in different locations, namely cortical, periventricular, and infratentorial regions; hence, DIR can be added to the MRI protocol of MS patients or even can replace FLAIR which would be of a good diagnostic value with only $80 \mathrm{~s}$ added to the scan time.
\end{abstract}

Keywords: Multiple sclerosis, MRI, FLAIR, DIR, Cortical lesions, White matter

\footnotetext{
* Correspondence: shaimafatooh@yahoo.com

1 Department of Radiology, Cairo University, Giza, Egypt

Full list of author information is available at the end of the article
}

\section{Springer Open}

(0) The Author(s). 2020 Open Access This article is licensed under a Creative Commons Attribution 4.0 International License, which permits use, sharing, adaptation, distribution and reproduction in any medium or format, as long as you give appropriate credit to the original author(s) and the source, provide a link to the Creative Commons licence, and indicate if changes were made. The images or other third party material in this article are included in the article's Creative Commons licence, unless indicated otherwise in a credit line to the material. If material is not included in the article's Creative Commons licence and your intended use is not permitted by statutory regulation or exceeds the permitted use, you will need to obtain permission directly from the copyright holder. To view a copy of this licence, visit http://creativecommons.org/licenses/by/4.0/. 


\section{Background}

Multiple sclerosis (MS) is one of the most common chronic inflammatory demyelinating diseases and a common cause of neurological disability in middle-aged and young adults. The main pathological hallmarks of the disease are demyelination, inflammation, and axonal damage, giving the characteristic multifocal lesions particularly targeting the periventricular, juxta cortical regions, brainstem, optic nerves, and spinal cord. However, considerable gray matter (GM) involvement is seen in many patients $[1,2]$.

Recent studies have claimed that the progression of the patient's disease course, the degree of physical disability, and the extent of cognitive impairment are closely associated with the degree of GM damage [2].

In January 2016, an updated version of the magnetic resonance imaging in multiple sclerosis (MAGNIMS) consensus MRI guidelines for the diagnosis of MS has been published, which further underlines the significance and importance of a proper assessment of cortical lesions (CL) and its potential role for the individual patient [3].

The diagnosis of MS and monitoring of disease progression are mainly based on MRI, which allows the establishment of early diagnosis within the specific diagnostic criteria. MR imaging for the diagnosis of MS is performed using a multisequence protocol including T2-weighted, fluid-attenuated inversion recovery (FLAIR), precontrast, and postcontrast T1-weighted sequences [4].

The pulse sequences show different sensitivities in the detection of inflammatory brain lesions depending on their anatomic location. FLAIR imaging provides the highest sensitivity in the detection of lesions close to the cerebrospinal fluid (CSF), such as the juxtacortical and the periventricular white matter, but is less sensitive in the posterior fossa. T2weighted sequences are known to be more sensitive in the detection of infratentorial lesions but have difficulties in detection of juxtacortical lesions. Unfortunately, no pulse sequence is available that provides a combination of a high sensitivity for the detection of supratentorial and infratentorial brain lesions in MS patients [5].

Although GM involvement was described in the earliest pathological studies of MS, conventional MRI sequences cannot reliably demonstrate cortical lesions owing to the small size of the lesions and lower contrast compared with surrounding normal appearing gray matter (NAGM) as well as partial volume effects of CSF in T2 and FLAIR images [5].

Few years ago, a new pulse sequence called double inversion recovery (DIR) was introduced. It provides two different inversion pulses (while FLAIR uses a single inversion pulse), which attenuates the CSF and the whole WM, thus achieving higher contrast and superior delineation between gray and white matter. The optimized contrast is due to differences in $\mathrm{T} 1$ relaxation times between GM/CSF and GM/WM $[6,7]$.
However, due to the long TI (inversion times) needed to suppress the CSF signal in both FLAIR and DIR, some artifacts may arise due to unsuppressed CSF flowing into the slice of interest from neighboring slices during the long TI interval, often mimicking pathology [7].

DIR may improve the visibility of GM lesions compared to other MRI sequences; however, the differentiation between different types of cortical lesions such as pure intracortical lesions, leucocortical lesions, and juxtacortical lesions is still challenging [6].

The aim of this study is to assess the role of DIR sequence in the detection of brain lesions in patients with MS compared to FLAIR sequence.

\section{Methods}

- A pilot study was performed on a convenient sample of 32 patients, having chronic relapsing remittent MS (RRMS), diagnosed according to McDonald criteria, referred from the Neurology to the Radiology Department at our institution during the period from August 2019 till February 2020. Among the thirty-two patients included in the study, there were 20 females and 12 males. The patients had a mean age of 30.75 \pm 8.6 years (ranged between $21-50$ years).

- Written informed consents were obtained from all patients. The study is IRB approved.

- All patients included in the study were subjected to: o Medical history assessment:

* Data was collected by reviewing medical records whenever possible as well as by direct patient interviewing. Thorough history taking was obtained including age, gender, occupation, diagnosis, age at onset and disease duration.

* Complete physical examination was performed for all patients by the neurologist (M.E) with 13 years experience.

o Inclusion criteria: RRMS patients diagnosed according to McDonald criteria coming for regular follow up and not suffering from acute exacerbation.

o Exclusion criteria: Patients with acute exacerbation of MS were excluded. Also patients with absolute contraindications to MRI as cardiac pacemakers and claustrophobic patients.

o Clinical assessment:

* Expanded Disability Status Scale (EDSS): The most frequently used clinical tool for assessment of the functional and clinical status of patients with MS. On a scale of 0 to 6 , functional system (FS) score measures how well the central nervous system is working and assigns a score to the disability. With the 
help of the FS score, the examiner rates the disability on the EDSS scale, the disability range includes 10 points describing the patient's efficiency from 0 (without disability), up to 10 (patient's death due to MS) [8].

* Cognitive and motor performance measures using the Symbol Digit Modalities Test (SDMT) which become the most commonly used neuropsychological test of cognitive assessment of MS patients in the last 5 years, due to its ease of administration and other factors such as reliability and validity, predictive validity, sensitivity, and specificity. The idea is measuring information processing speed and efficiency using a symbol/digit substitution task [9].

o Magnetic resonance imaging:

* All patients were scheduled for brain MRI in Radiology Department, using 1.5 Tesla superconducting magnet Philips MRI machine, Achieva, Netherland.

* The following sequences were obtained:

- Conventional MRI sequences: axial T1WI, axial T2WI, axial FLAIR, sagittal T1WI, coronal T1WI, diffusion-weighted imaging.

- Sagittal 3D FLAIR.

- Sagittal 3D DIR.

* All patients were examined in the supine position, head first.

* Acquisition parameters:

- T1WI: TR $450 \mathrm{~ms}$, TE $15 \mathrm{~ms}$, matrix $208 \times 134$, FOV $230 \times 177 \mathrm{~mm}^{2}$, slice thickness $6 \mathrm{~mm}$.

- T2WI: TR 3612 ms, TE 100 ms, matrix $208 \times 127$, FOV $230 \times 177 \mathrm{~mm}^{2}$, slice thickness $6 \mathrm{~mm}$.

- FLAIR: TR 6000 ms, TE 120 ms, TI 2000 ms, matrix $240 \times 111$, FOV $230 \times 184$ $\mathrm{mm}^{2}$, slice thickness $6 \mathrm{~mm}$.

- Scan time of conventional MRI: 7 min $52 \mathrm{~s}$.

- 3D sagittal FLAIR: TR 4800 ms, TE 270 ms, TI $1660 \mathrm{~ms}$, turbo factor 178, matrix 256, FOV $230 \times 123 \mathrm{~mm}^{2}$, voxel size $1.5 \times 1.5 \times$ $0.56 \mathrm{~mm}^{3}$, NSA 3 , scan time $3 \mathrm{~min} 26 \mathrm{~s}$.

- 3D sagittal DIR: TR $5500 \mathrm{~ms}$, TE $273 \mathrm{~ms}$, TI1 $2510 \mathrm{~ms}$, TI2 $480 \mathrm{~ms}$, turbo factor 208, matrix 256, FOV $230 \times 123 \mathrm{~mm}^{2}$, voxel size $1.5 \times 1.5 \times 0.56 \mathrm{~mm}^{3}$, NSA 3, scan time 4 $\min 46 \mathrm{~s}$.

* The FLAIR and DIR sequences were obtained with identical anatomic position, using same FOV, matrix, voxel size, and NSA.

* DIR and FLAIR were viewed by three radiologists (R.E, S.F and M.A) with 30, 14, and 3 years experience, blinded to the outcome of the other sequence and the clinical features of the patients.

* MS lesions appeared as hyper-intense spots with a size of $\geq 3 \mathrm{~mm}$. Contrary to these hyperintense spots, there was some high signal intensity in a striped shape known as flow artifacts in extra

cortical regions rising from the sinuses or major vessels.

* The lesions were counted and classified according to 5 anatomic regions:

- Infratentorial lesions.

- Periventricular lesions which are adjacent to the lateral ventricles.

- Deep white matter lesions which are in deep white matter.

- Juxtacortical lesions which actually are in the white matter but touch the cortex.

- Cortical lesions with no visual affection of the juxtacortical or subcortical cerebral white matter.

In addition to:

- Corpus callosum lesions.

- Craniocervical junction lesions.

\section{Statistical analysis}

- Data were coded and entered using the statistical package for the Social Sciences (SPSS) version 25 (IBM Corp., Armonk, NY, USA). Data was summarized using mean, standard deviation, median, minimum and maximum in quantitative data, and using frequency (count) and relative frequency (percentage) for categorical data.

- Comparisons between quantitative variables were done using the non-parametric Mann-Whitney test. For comparison of paired measurements within each patient, the non-parametric Wilcoxon signed rank test was used. Correlations between quantitative variables were done using Spearman correlation coefficient. $P$ values less than 0.05 were considered as statistically significant.

\section{Results}

\section{Clinical data}

The patients had a mean age at onset of 25.41 years (range 10-45 years), and a mean disease duration of 5.34 years (range 1-14 years). The patients had a mean expanded disability status scale (EDSS) of 2.48 (range 0-7) and a mean symbol digit modalities test of 25.44 (range 17-38) (Table 1). 
Table 1 Clinical data of patients

\begin{tabular}{llllll}
\hline & Mean & SD & Median & Minimum & Maximum \\
\hline Age at onset & 25.41 & 8.12 & 22.00 & 10.00 & 45.00 \\
Disease duration & 5.34 & 3.95 & 4.50 & 1.00 & 14.00 \\
EDSS & 2.48 & 1.56 & 2.50 & 0.00 & 7.00 \\
Symbol digit modalities test & 25.44 & 5.83 & 24.00 & 17.00 & 38.00 \\
\hline
\end{tabular}

\section{Comparison between DIR and FLAIR sequences at different brain regions}

DIR showed a significantly higher total number of MS lesions in the 32 patients included in the study (total of 1690 lesions were detected in DIR compared to 1469 lesions in FLAIR) (Table 2 and Figs. 1, 2, 3 and 4). The average number of lesions per patient was $52.81 \pm 1.71$ in DIR and $45.9 \pm 1.42$ in FLAIR with a statistically significant difference $(p<0.001)$. Regarding the infratentorial lesions, DIR detected higher number of lesions compared to FLAIR (total number of lesions was 93 in DIR and 72 in FLAIR while average number of lesions was $2.91 \pm 2.66$ in DIR compared to $2.25 \pm 2.18$ in FLAI $\mathrm{R})$ with a statistically significant difference $(p=0.002)$. Also in supratentorial periventricular regions (total number of lesions was 379 in DIR and 362 in FLAIR while average number of lesions was $11.84 \pm 8.07$ in DIR compared to $11.31 \pm 8.07$ in FLAIR) with a statistically significant difference $(p>0.001)$. DIR imaging also demonstrated significantly more intracortical lesions (total 228 lesions with average of $7.13 \pm 7.19)$ compared to FLAIR imaging (total of 45 lesions with $1.41 \pm 1.76$ ) with a statistically significant difference $(p<0.001)$. On the other hand, corpus callosum lesions were significantly higher on FLAIR (total of 27 lesions, average was $0.84 \pm$ 1.25) with respect to DIR imaging (total was 22 lesions with an average of $0.69 \pm 1.12$ ) with a statistically significant difference in between $(p=0.025)$. Regarding the juxtacortical lesions, DIR detected higher number of lesions than FLAIR (4.91 \pm 5.4 compared to $4.72 \pm 5.3$ ) although not statistically significant $(p=0.102)$.

\section{Correlation between clinical and radiological data}

There was a statistically significant positive correlation between patient's age and number of cortical lesions in FLAIR $(p=0.002)$ and DIR $(p=0.002)$. On the other hand, there was a statistically significant negative correlation between age at onset and number of deep white matter lesions in FLAIR $(p=0.005)$ and DIR ( $p=0.005)$ (Table 3$)$. As regard disease duration, there was a statistically significant positive correlation between disease duration and number of periventricular $(p=0.001$ for FLAIR and 0.001 for DIR), deep white matter ( $p=0.033$ for FLAIR and 0.033 for DIR), juxtacortical ( $p=0.018$ for FLAIR and 0.035 for DIR), and cortical ( $p=0.009$ for FLAIR and 0.002 for DIR) lesions in FLAIR and DIR.

There was a positive correlation between EDSS and number of periventricular $(p=0.483$ for FLAIR and 0.555 for DIR), deep white matter ( $p=0.797$ for FLAIR and 0.797 for DIR), infratentorial ( $p=0.522$ for FLAIR and 0.355 for DIR), and corpus callosum ( $p=0.732$ for FLAIR and 0.947 for DIR) lesions in FLAIR and DIR, although not statistically significant.

There was a negative correlation between symbol digit modalities test and number of juxtacortical $(p=0.653$ for FLAIR and 0.731 for DIR) and cortical ( $p=0.512$ for FLAIR and 0.392 for DIR) lesions in FLAIR and DIR although not statistically significant.

\section{Discussion}

Multiple sclerosis is a common chronic inflammatory disease and a common causes of disability in young

Table 2 Comparison between DIR and FLAIR regarding the number of lesions, mean, and SD in the 32 patients

\begin{tabular}{|c|c|c|c|c|c|c|c|}
\hline & \multicolumn{3}{|l|}{ FLAIR } & \multicolumn{3}{|l|}{ DIR } & \multirow[t]{2}{*}{$P$ value } \\
\hline & $\overline{\text { No. }}$ & Mean & SD & $\overline{\text { No. }}$ & Mean & SD & \\
\hline Total number of lesions in all patients & 1469 & 45.9 & 1.42 & 1690 & 52.81 & 1.71 & $<0.001$ \\
\hline Number of periventricular lesions & 362 & 11.31 & 8.07 & 379 & 11.84 & 8.07 & $<0.001$ \\
\hline Number of deep white matter lesions & 802 & 25.06 & 22.01 & 802 & 25.06 & 22.01 & 1 \\
\hline Number of juxtacortical lesions & 151 & 4.72 & 5.30 & 157 & 4.91 & 5.43 & 0.102 \\
\hline Number of cortical lesions & 45 & 1.41 & 1.76 & 228 & 7.13 & 7.19 & $<0.001$ \\
\hline Number of infratentorial lesions & 72 & 2.25 & 2.18 & 93 & 2.91 & 2.66 & 0.002 \\
\hline Number of cranio-cervical junction lesions & 10 & 0.31 & 0.64 & 9 & 0.28 & 0.63 & 0.317 \\
\hline Number of corpus callosum lesions & 27 & 0.84 & 1.25 & 22 & 0.69 & 1.12 & 0.025 \\
\hline
\end{tabular}




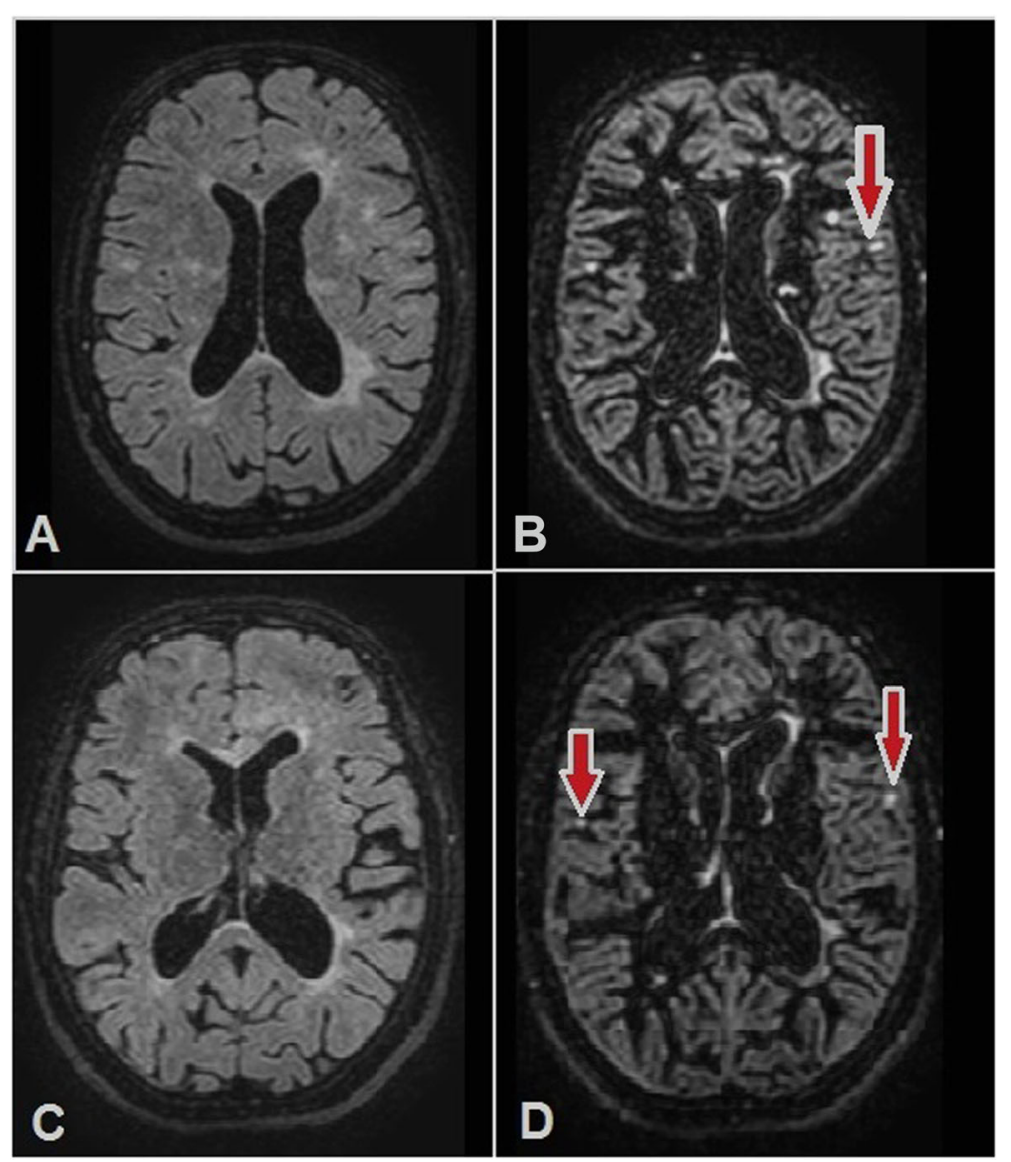

Fig. 1 A 20-year-old female patient with MS and recurrent bouts of clinical symptoms (attacks of blurring of vision), with EDSS score of 2.5 and SDMT of 21. a Axial FLAIR showing irregular patchy areas and foci (plaques) of abnormal white matter high FLAIR and T2 signal intensity are seen implicating both cerebral periventricular and subcortical regions. b Axial DIR at the same level showing a left frontal cortical lesion being hardly identified on FLAIR imaging. c Axial FLAIR and $\mathbf{d}$ axial DIR at the same level of (c) showing left insular cortical lesion as well as right insular juxtacortical lesions are detected on DIR. The total number of cortical lesions detected in this case was ( 9 for DIR and 2 for FLAIR) and juxtacortical lesions (5 for DIR and 3 for FLAIR)

adults. Traditionally, MS was considered to be caused by demyelination in white matter; however, the affection of gray matter is becoming increasingly prominent. Grey matter lesions have even been reported to precede white matter demyelination with some recent studies indicate an association of cortical lesion with clinical and cognitive impairment in MS patients [10,11].

The diagnosis of MS is based mainly on MR imaging with conventional multisequence protocols. Owing to its ability to attenuate the CSF signal, FLAIR imaging is highly sensitive in detection of supratentorial lesions, especially juxtacortical and periventricular white matter lesions. Double inversion recovery (DIR) imaging technique uses a combination of 2 inversion pulses providing a sufficient attenuation of both CSF and the normal appearing white matter [7].

This study aims to investigate whether or not the 3D DIR sequence is superior compared to the 3D FLAIR sequence in detecting brain lesions in MS patients. The correlations between lesion load and disability status and cognitive functions in these patients were also investigated in this study.

DIR showed a significantly higher total number of MS lesions compared to FLAIR (total of 1690 lesions were detected in DIR compared to 1469 lesions in FLAIR), conforming to the study done over 55 patients with MS by Abidi Z. et al [5]. They found a significantly higher total number of lesions were displayed on DIR 2658 lesions while 2513 lesions on FLAIR images $(p=0.000)$. Also our results agreed with another study performed over 15 MS patients by Elnekeidy AM et al. [12] who found 349 lesions detected by DIR sequence compared to 267 lesions detected by FLAIR.

In our study, more infratentorial lesions were observed on DIR with an average of $2.91 \pm 2.66$ compared to $2.25 \pm$ 2.18 in FLAIR; also, we found that DIR has higher accuracy to detect intracortical lesions with respect to FLAIR sequence (average of $7.13 \pm 7.19$ in DIR 


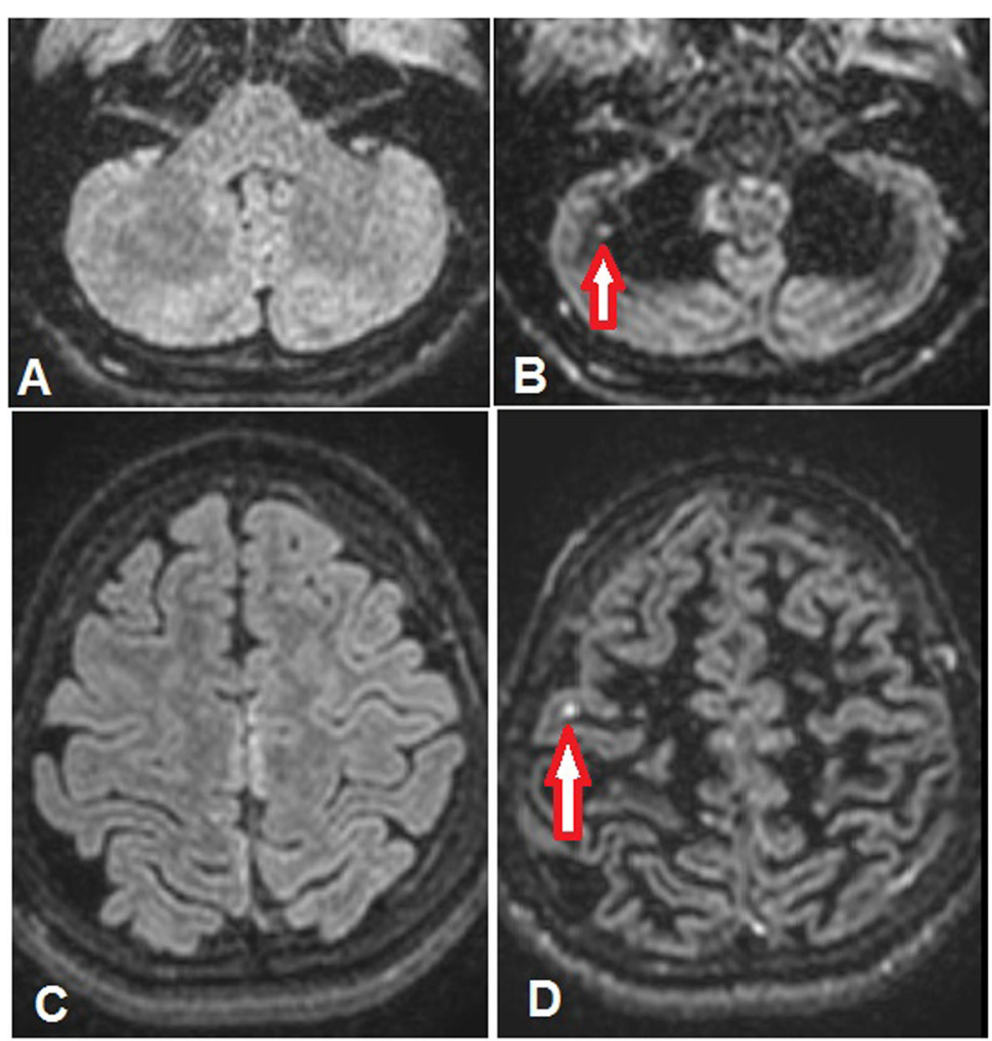

Fig. 2 A 29-year-old female patient with MS and EDSS score of 1 and SDMT of 34. a Axial FLAIR and $\mathbf{b}$ axial DIR at the same level showing a right cerebellar lesion being not identified on FLAIR imaging. c Axial FLAIR and $\mathbf{d}$ axial DIR at the same level showing a right frontal juxtacortical lesion detected on DIR being not seen in FLAIR images. The total number of juxtacortical lesions detected in this case was (10 for DIR and 8 for FLAIR) and infratentorial lesions (5 for DIR and 3 for FLAIR)

compared to $1.41 \pm 1.76$ in FLAIR). Our results were equivalent to previous studies such as Abidi Z. et al [5] who detected an average of $5.1 \pm 7.37$ infratentorial lesions in DIR compared to $4.23 \pm 6.45$ in FLAIR with $P$ 0.000 and $1.29 \pm 1.04$ intracortical lesions in DIR compared to $0.5 \pm 0.71$ in FLAIR with a $P 0.000$. Also we agreed with the results of Elnekeidy AM et al. [12] who found an average of $8.21 \pm 9.99$ infratentorial lesions in DIR compared to $2.07 \pm 3.41$ in FLAIR $(P$ 0.001) and an average of $6.57 \pm 7.44$ intracortical lesions in DIR compared to $2.86 \pm 3.88$ in FLAIR $(P$ 0.003). Our results also conform with Vural G. et al. [13] who studied 34 patients with MS and found that DIR was superior to FLAIR in detection of infratentorial and cortical lesions with an average of $0.8 \pm 1.2$ infratentorial lesions in DIR compared to $0.7 \pm 0.8$ in FLAIR and $10.1 \pm 7.4$ intracortical lesions in DIR compared to $2.7 \pm 3.1$ in FLAIR.

A higher number of juxtacortical white matter lesions were detected on DIR compared to FLAIR (4.91 \pm 5.4 compared to $4.72 \pm 5.3$ ), although not statistically significant ( $P$ 0.102). Abidi et al. [5] found that DIR detected higher number of lesions than FLAIR which agreed with our results yet with a significant statistical difference $(15.20 \pm 8.61$ compared to $14.07 \pm 8.11$ with $P$ 0.000). Also Elnekeidy et al. [12] found higher number of lesions in DIR with a significant statistical difference $(P$ 0.002). On the other hand, Geurts et al. [14] reported the highest number of juxtacortical lesions on T2weighted images. Our results also disagreed with Wattjes et al. [15] who studied seventeen patients presenting with a clinically isolated syndrome (CIS) suggestive of MS and they found higher number of juxtacortical lesions in FLAIR compared to DIR (total of 57 compared to 54 lesions respectively with insignificant statistical difference) $(P$ 0.08). Such disagreement could be related to the lower number of patients in their study.

In the present study, DIR sequence demonstrated a higher number of periventricular WM lesions (11.84 \pm 8.07) compared to $11.31 \pm 8.07$ in FLAIR with a statistically significant difference $p<0.001$ which agreed with Elnekeidy et al. [12] who found DIR superior to FLAIR with a significant statistical difference $(P$ 0.038). Our results also agreed to some extent with Abidi et al. [5] who found the average lesions in DIR was $15.34 \pm 11.34$ compared to $15.18 \pm$ 

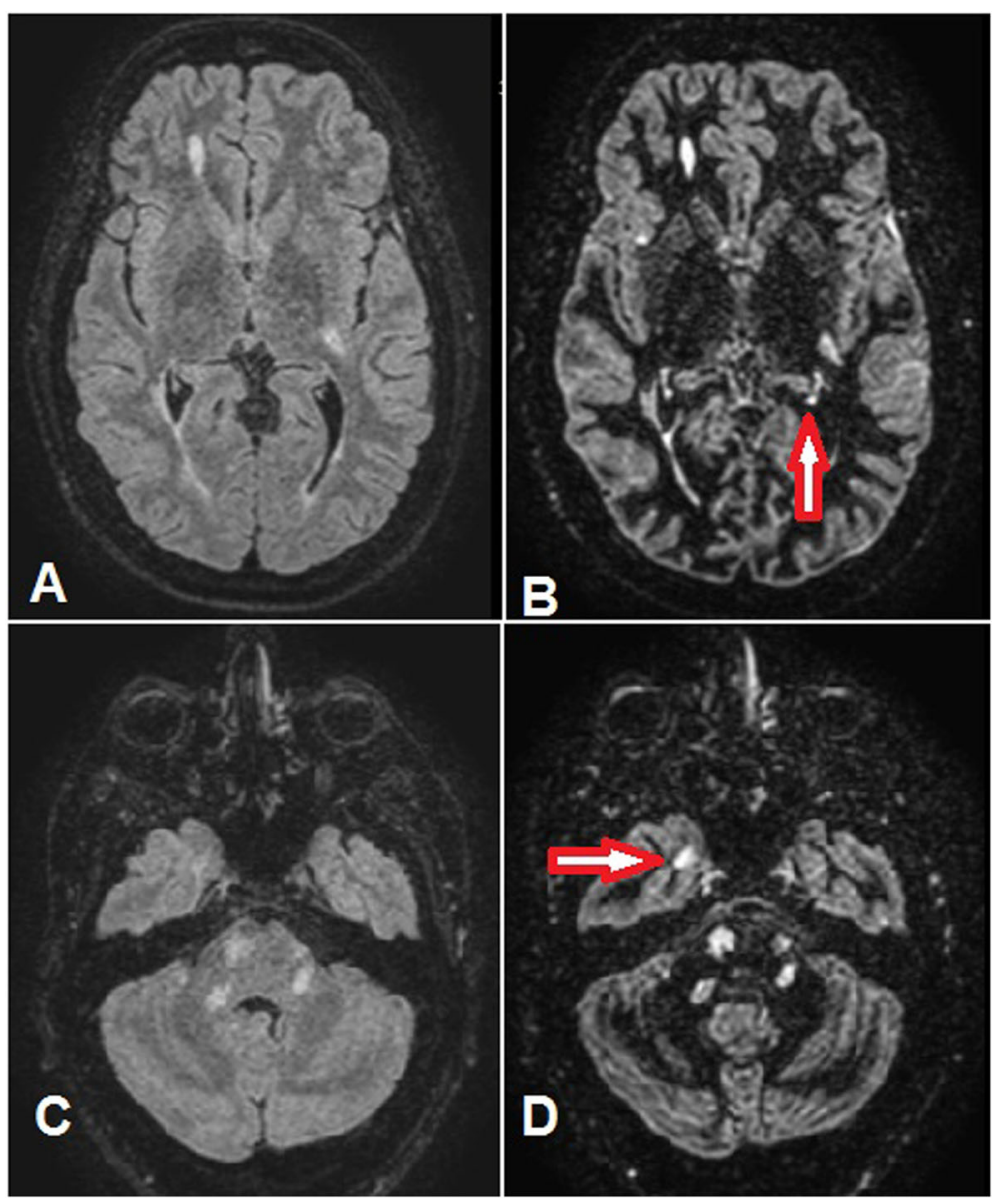

Fig. 3 A 32-year-old male patient with MS and EDSS score of 2 and SDMT of 27. a Axial FLAIR and $\mathbf{b}$ axial DIR at the same level showing a small left deep periventricular focus not well seen in the FLAIR images (arrowed). c Axial FLAIR and $\mathbf{d}$ axial DIR at the same level showing a right temporal cortical lesion detected on DIR which could be easily missed in FLAIR images. The other lesions seen in the pons and middle cerebellar peduncles are well identified in both sequences. The total number of cortical lesions detected in this case was ( 6 for DIR and 2 for FLAIR) and periventricular lesions (15 for DIR and 13 for FLAIR)

11.16 in FLAIR yet with insignificant statistical difference $(P$ 0.14) and also with Wattjes et al. [15] who found a total of 85 lesions in DIR compared to 81 lesions in FLAIR with insignificant statistical difference $P 0.16$.

An equal number of MS lesions were observed in deep white matter in the DIR and FLAIR sequences. However, Vural et al. [13] reported a higher number of detected lesions in deep white matter with T2W and FLAIR imaging compared to DIR $(p=0.022$ and $p=0.027$, respectively).

MS lesions near the CSF were more countable on DIR compared with FLAIR. This could be explained by the better contrast obtained by suppression of CSF on DIR images. Also the distinction between normal appearing gray matter/normal appearing white matter is markedly improved at DIR images; so, deciding about the location of the lesion in GM or WM was easier compared with FLAIR [5].
Flow artifacts observed in DIR images is seen in the posterior fossa, periventricular white matter, choroid plexus, and periaqueductal region of the brainstem tissue, which may be from CSF pulsation or from sinuses and bigger vessels. Another high signal ribbon-like artifact in the phase direction was often observed in extra-cortical regions. Also, lower SNR in the DIR technique and inhomogeneity of the magnetic field in the cortex of the limbic lobe and diminished inhomogeneity in the cortex of central sulcus should be noticed. Viewing multiple slices and other MRI sequences such as T1-weighted or FLAIR will help to report them as lesions (which are in an irregular shape) or as an artifact resulting from cortical vessels, which is rounded [5]. However, we did not face this artifact to a degree affecting the interpretation of images and it has no impact on our results.

Correlation between clinical and radiological data showed a positive correlation between EDSS and number 


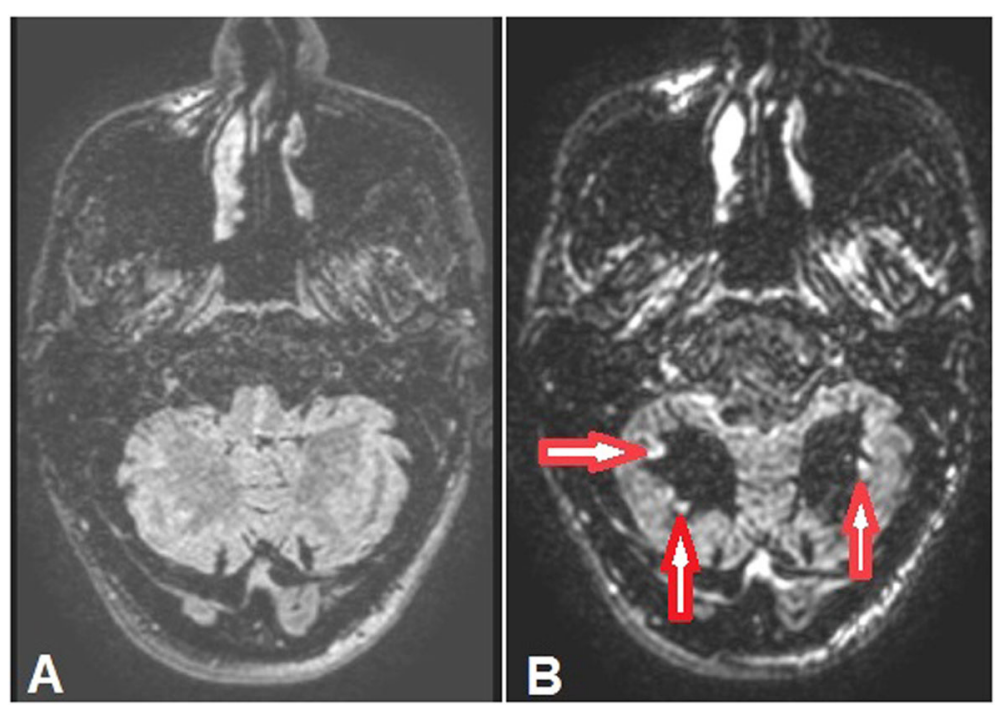

Fig. 4 A 44-year-old female patient male patient with MS and EDSS score of 3.5 and SDMT of 20. a Axial FLAIR and $\mathbf{b}$ Axial DIR at the same level showing bilateral cerebellar foci of high signal being more easily identified in DIR rather than in FLAIR images. The total number of infratentorial lesions detected in this case was (7 for DIR and 4 for FLAIR)

of periventricular lesions, deep white matter lesions, and infratentorial lesions in both FLAIR and DIR, although not statistically significant (as detailed in Table 3). However, in the previous study done by Calabrese et al. [16], over 380 patients of MS (clinically isolated syndrome, relapsing-remitting MS, and secondary progressive MS) found a significant positive correlation between the number of intracortical lesions and EDDS scores which is an indicator for disability ( $P$ 0.004).

Ertan et al. 2018 showed that EDSS scores were higher in patients with long disease duration and increased number of intracortical lesions. However, no significant correlation was found between lesion load and the EDSS scores in all regions which is compatible with the results of our study [17].

Studying the correlation between lesion load and cognitive impairment, there was a non-significant negative correlation between the lesion load in FLAIR $(p=0.512$ for cortical lesions) and DIR ( $p=0.392$ for cortical lesions) sequences and the results of SDMT cognitive assessment. The explanation of such weak correlation could be related to the relatively small number of cases and also that subpial cortical lesions, which are associated with physical and cognitive dysfunction in patients with MS, are poorly detected by conventional MRI and even DIR [15].

Table 3 Correlation between clinical and radiological data

\begin{tabular}{|c|c|c|c|c|c|c|}
\hline & & Age & Age at onset & Disease duration & EDSS score & $\begin{array}{l}\text { Symbol digit } \\
\text { modalities test }\end{array}$ \\
\hline Number of periventricular lesions in FLAIR & $P$ value & 0.355 & 0.442 & 0.001 & 0.483 & 0.281 \\
\hline Number of periventricular lesions in DIR & $P$ value & 0.333 & 0.512 & 0.001 & 0.555 & 0.305 \\
\hline Number of deep white matter lesions in FLAIR & $P$ value & 0.271 & 0.005 & 0.033 & 0.797 & 0.245 \\
\hline Number of deep white matter lesions in DIR & $P$ value & 0.271 & 0.005 & 0.033 & 0.797 & 0.245 \\
\hline Number of juxtacortical lesions in FLAIR & $P$ value & 0.567 & 0.384 & 0.018 & 0.983 & 0.653 \\
\hline Number of juxtacortical lesions in DIR & $P$ value & 0.656 & 0.370 & 0.035 & 0.870 & 0.731 \\
\hline Number of cortical lesions in FLAIR & $P$ value & 0.002 & 0.046 & 0.009 & 0.489 & 0.512 \\
\hline Number of cortical lesions in DIR & $P$ value & 0.002 & 0.066 & 0.002 & 0.173 & 0.392 \\
\hline Number of infratentorial lesions in FLAIR & $P$ value & 0.868 & 0.485 & 0.130 & 0.522 & 0.212 \\
\hline Number of infratentorial lesions in DIR & $P$ value & 0.818 & 0.493 & 0.117 & 0.355 & 0.105 \\
\hline
\end{tabular}


The study has some limitations including the relatively small sample size, measurement of the number of the lesions only discarding the lesion's volume, even though several studies reported cortical lesions volume as a more accurate predicting factor of cognitive impairment. However, volumetric measures are usually technically difficult, while cortical lesions count can be easily evaluated. Some studies reported that DIR is a timeconsuming sequence and has a high tissue absorption rate. Based on such disadvantages over FLAIR, one might think that its routine use in MS imaging may not be justified [18, 19]; however, in our study, 3D DIR sequence required an additional $4 \mathrm{~min} 46 \mathrm{~s}$ only. The aim of our study was to compare the number of detected lesions on FLAIR and DIR sequences. Thus, it was performed without intravenous contrast administration, so we cannot judge the role of DIR in active MS lesions which needs further larger studies comparing post contrast T1 WIs with DIR and FLAIR images.

\section{Conclusion}

DIR is a powerful conventional MRI sequence for visualization of brain lesions in patients with MS and is superior to FLAIR sequence in detecting lesions in different locations, namely cortical, periventricular, and infratentorial regions; hence and based upon our results, DIR can be added to the MRI protocol of MS patients or even can replace FLAIR which would be of a good diagnostic value with only $80 \mathrm{~s}$ added to the scan time.

\section{Abbreviations \\ CL: Cortical lesions; CSF: Cerebrospinal fluid; DIR: Double inversion recovery; EDSS: Expanded Disability Status Scale; FLAIR: Fluid attenuation inversion recovery; GM: Grey matter; MAGNIMS: Magnetic resonance imaging in multiple sclerosis; MS: Multiple sclerosis; NAGM: Normal appearing gray matter; RRMS: Relapsing remittent multiple sclerosis; SDMT: Symbol Digit Modalities Test; WM: White matter}

\section{Acknowledgements}

Not applicable.

\section{Authors' contributions}

S.F.E put the study design, editor of the manuscript, and performed the MRI assessment. M.A.S participation in the MRI assessment, data collection, and performed statistical analysis. M.E.M data collection and clinical assessment of the patients. R.E.A put the idea of the study, participated in the study design, and MRI assessment. All authors read and approved the final manuscript.

\section{Funding}

Not applicable (no funding)

\section{Availability of data and materials}

All the datasets used and analyzed in this study are available with the corresponding author on reasonable request.

\section{Ethics approval and consent to participate}

Written informed consent was signed by all patients before the MRI examination. The study is approved by the medical committee of the faculty of medicine Cairo university at March 2019. Reference number not available.

\section{Consent for publication}

All patients included in this research were older than 18 years old and gave written informed consent to publish the data contained within this study.

\section{Competing interests}

The authors declare that they have no financial or nonfinancial competing interest.

\section{Author details}

'Department of Radiology, Cairo University, Giza, Egypt. ${ }^{2}$ Neurology Department, Cairo University, Giza, Egypt.

Received: 29 April 2020 Accepted: 17 August 2020

Published online: 15 September 2020

\section{References}

1. Hassan TA, Elkholy SF, Mahmoud BE, ElSherbiny M (2019) Multiple sclerosis and depressive manifestations: can diffusion tensor MR imaging help in the detection of microstructural white matter changes? Egypt J Radiol Nucl Med 50(1):31

2. Faizy TD, Thaler C, Ceyrowski T, Broocks G, Treffler N, Sedlacik J, Siemonsen S (2017) Reliability of cortical lesion detection on double inversion recovery MRI applying the MAGNIMS-Criteria in multiple sclerosis patients within a 16-months period. PLoS One 12(2): e0172923

3. Filippi M, Rocca MA, Ciccarelli O, De Stefano N, Evangelou N, Kappos L, Gasperini C (2016) MRI criteria for the diagnosis of multiple sclerosis: MAGN IMS consensus guidelines. Lancet Neurol 15(3):292-303

4. Hemond CC, Bakshi R (2018) Magnetic resonance imaging in multiple sclerosis. Cold Spring Harb Perspect Med 8(5):a028969 https://doi.org/10. 1101/cshperspect.a028969

5. Abidi Z, Faeghi F, Mardanshahi Z, Mortazavi H (2017) Assessment of the diagnostic accuracy of double inversion recovery sequence compared with FLAIR and T2W_TSE in detection of cerebral multiple sclerosis lesions. Electron Physician 9(4):4162

6. Favaretto A, Poggiali D, Lazzarotto A, Rolma G, Causin F, Gallo P (2015) The parallel analysis of phase sensitive inversion recovery (PSIR) and double inversion recovery (DIR) images significantly improves the detection of cortical lesions in multiple sclerosis (MS) since clinical onset. PLoS One 10(5):e0127805

7. Saranathan M, Worters PW, Rettmann DW, Winegar B, Becker J (2017) Physics for clinicians: fluid-attenuated inversion recovery (FLAIR) and double inversion recovery (DIR) Imaging. J Magn Reson Imaging 46(6): 1590-1600

8. Kucharczuk M, Juraszek KM, Krajewski S, Surman-Wika M, Tkaczyński K (2018) The assessment of the patients disability degree using the EDSS scale in various forms of multiple sclerosis. J Educ Health Sport 8(3): 278-292

9. Benedict RH, DeLuca J, Phillips G, LaRocca N, Hudson LD, Rudick R, Multiple Sclerosis Outcome Assessments Consortium (2017) Validity of the symbol digit modalities test as a cognition performance outcome measure for multiple sclerosis. Mult Scler J 23(5):721-733

10. Kolber P, Montag S, Fleischer V, Luessi F, Wilting J, Gawehn J, Gröger A, Zipp F (2015) Identification of cortical lesions using DIR and FLAIR in early stages of multiple sclerosis. J Neurol 262(6):1473-1482

11. Harrison DM, Roy S, Oh J, Izbudak I, Pham D, Courtney S, Caffo B, Jones CK, van Zijl P, Calabresi PA (2015) Association of cortical lesion burden on 7-T magnetic resonance imaging with cognition and disability in multiple sclerosis. JAMA Neurol 72(9):1004-1012

12. Elnekeidy AM, Kamal MA, Elfatatry AM, Elskeikh ML (2014) Added value of double inversion recovery magnetic resonance sequence in detection of cortical and white matter brain lesions in multiple sclerosis. Egypt J Radiol Nucl Med 45(4):1193-1199

13. Vural G, Keklikoğlu HD, Temel ş, Deniz O, Ercan K (2013) Comparison of double inversion recovery and conventional magnetic resonance brain imaging in patients with multiple sclerosis and relations with disease disability. Neuroradiol J 26(2):133-142

14. Wattjes MP, Lutterbey GG, Gieseke J, Träber F, Klotz L, Schmidt S, Schild $\mathrm{HH}$ (2007) Double inversion recovery brain imaging at 3T: diagnostic value in the detection of multiple sclerosis lesions. Am J Neuroradiol 28(1):54-59 
15. Geurts JJ, Roosendaal SD, Calabrese M, Ciccarelli O, Agosta F, Chard DT, Gass A, Huerga E, Moraal B, Pareto D, Rocca MA (2011) Consensus recommendations for MS cortical lesion scoring using double inversion recovery MRI. Neurology 76(5):418-424

16. Calabrese M, De Stefano N, Atzori M, Bernardi V, Mattisi I, Barachino L, Morra A, Rinaldi L, Romualdi C, Perini P, Battistin L (2007) Detection of cortical inflammatory lesions by double inversion recovery magnetic resonance imaging in patients with multiple sclerosis. Arch Neurol 64(10):1416-1422

17. Ertan G, Düz Arıı Ö, Ulus S, Metin B (2018) Efficiency of double ınversion recovery (dir) sequence in the evaluation of supratentorial cortical lesions in multiple sclerosis. J Neuroquantol 16(3):23-29

18. Damasceno A, Damasceno BP, Cendes F (2015) Subclinical MRI disease activity influences cognitive performance in MS patients. Mult Scler Relat Disord 4(2):137-143

19. Papadopoulou A, Müller-Lenke N, Naegelin Y, Kalt G, Bendfeldt K, Kuster P, Stoecklin M, Gass A, Sprenger T, Radue EW, Kappos L (2013) Contribution of cortical and white matter lesions to cognitive impairment in multiple sclerosis. Mult Scler J 19(10):1290-1296

\section{Publisher's Note}

Springer Nature remains neutral with regard to jurisdictional claims in published maps and institutional affiliations.

\section{Submit your manuscript to a SpringerOpen ${ }^{\circ}$ journal and benefit from:}

- Convenient online submission

Rigorous peer review

- Open access: articles freely available online

- High visibility within the field

- Retaining the copyright to your article

Submit your next manuscript at $\boldsymbol{\nabla}$ springeropen.com 